DOI: 10.1007/s00350-016-4431-x

\section{Kein Vorrang der Leistungsklage bei fortbestehenden Personenschäden}

ZPO §265

Der Kläger ist nicht gehalten, seine Klage in eine Leistungs- und in eine Feststellungsklage aufzuspalten, wenn bei Klageerhebung ein Teil des Schadens schon entstanden, die Entstehung weiteren Schadens aber noch zu erwarten ist. Einzelne bei Klageerhebung bereits entstandene Schadenspositionen stellen lediglich einen Schadensteil in diesem Sinne dar.

BGH, Urt. v. 19. 4.2016 - VI ZR 506/14 (OLG Oldenburg)

Problemstellung: Der BGH stellt klar, dass im Falle einer noch nicht abgeschlossenen Schadensentwicklung, wie sie im Zusammenhang mit der Geltendmachung folgenschwerer Behandlungsfehler typischerweise vorliegt, der Kläger seine Klage nicht aufspalten muss, um bereits bezifferbare Schadensposten mit der Leistungsklage $\mathrm{zu}$ verfolgen, er vielmehr in vollem Umfang Feststellung der Ersatzpflicht verlangen kann (s. auch BGH, NJW 1984, 1118; zur Arzthaftung OLG Koblenz, MedR 2010, 507 f.; zur Klage im Einzelnen Wenzel, in: ders. [Hrsg.], Handbuch des Fachanwalts Medizinrecht, 3. Aufl. 2013, Kap. 7, Rdnrn. 260 ff.). Das erforderliche Feststellungsinteresse i. S. des $\$ 256$ Abs. $1 \mathrm{ZPO}$ ist gegeben, wenn ,die Durchführung des Feststellungsverfahrens unter dem Gesichtspunkt der Prozesswirtschaftlichkeit zu einer sinnvollen und sachgemäßen Erledigung der aufgetretenen Streitpunkte führt". Indem der BGH die Darlegungslast reduziert und die Bezifferung der Schäden einem Folgeprozess oder einer nachprozessualen vergleichsweisen Lösung überlässt, erleichtert er dem Patienten die Rechtsverfolgung.

Zum Sachverhalt: Der Kl. nimmt die Bekl. wegen einer bei seiner nicht ausreichend aufgeklärten Mutter in der 34. Schwangerschaftswoche rechtswidrig vorgenommenen sectio, die bei ihm zu einer Schwerstbehinderung geführt hat, auf Schmerzensgeld und Feststellung in Anspruch.

Das LG hat die Klage abgewiesen. Das OLG hat durch TeilGrund- und Teil-Endurteil entschieden, dass der auf Zahlung von Schmerzensgeld gerichtete Klageantrag dem Grunde nach gerechtfertigt ist. Insoweit hat es die Sache zur Verhandlung und Entscheidung über die Höhe des Schmerzensgeldes an das LG zurückverwiesen. Es hat ferner festgestellt, dass die Bekl. verpflichtet ist, dem Kl. sämtliche im Zeitpunkt der Klageerhebung noch nicht bezifferbaren oder in der Fortentwicklung befindlichen sowie zukünftigen materiellen Schäden zu ersetzen, die ihm durch die rechtswidrige Kaiserschnittentbindung entstanden sind oder entstehen werden, soweit die Ansprüche nicht auf Sozialversicherungsträger oder sonstige Dritte übergegangen sind oder übergehen werden. Im Übrigen hat es wegen des weitergehenden Feststellungsantrags die Klage abgewiesen und die Berufung des Kl. zurückgewiesen.

Mit der vom Senat zugelassenen Revision verfolgte der Kl. sein Feststellungsbegehren, soweit das Berufungsgericht ihm nicht bereits entsprochen hat, weiter.

Aus den Gründen: [4] I. Das Berufungsgericht hat zur Begründung seiner Entscheidung - soweit hier erheblich im Wesentlichen ausgeführt, es sei klarzustellen, dass nur

Eingesandt vom BGH-Entscheidungsversand, Karlsruhe; bearbeitet von Prof. Dr. iur. Christian Katzenmeier, Institut für Medizinrecht, Universität zu Köln, Albertus-Magnus-Platz, 50923 Köln, Deutschland solche materiellen Schäden umfasst seien, die zur Zeit der Klageerhebung nicht bezifferbar gewesen seien oder sich noch in der Fortentwicklung befunden hätten. Dass im Fall des Kl. zukünftige oder in der Fortentwicklung befindliche Schäden möglich seien, liege angesichts der erlittenen Hirnschädigung auf der Hand. Mit Blick auf Schäden, die bereits bei Klageerhebung bezifferbar gewesen seien und sich nicht in der Fortentwicklung befunden hätten, fehle es dagegen an dem notwendigen Feststellungsinteresse. Insoweit sei der Feststellungsantrag des Kl. wegen des Vorrangs der Leistungsklage unzulässig.

[5] II. Das Berufungsurteil hält der revisionsrechtlichen Überprüfung in einem entscheidenden Punkt nicht stand. Das Berufungsgericht hat ein rechtliches Interesse $(\$ 256$ Abs. 1 ZPO) des Kl. an der weitergehenden Feststellung hinsichtlich des bei Klageerhebung bereits bezifferbaren Schadensteils zu Unrecht verneint.

[6] 1. Es ist anerkannt, dass der Kläger grundsätzlich nicht gehalten ist, seine Klage in eine Leistungs- und in eine Feststellungsklage aufzuspalten, wenn bei Klageerhebung ein Teil des Schadens schon entstanden, die Entstehung weiteren Schadens aber noch zu erwarten ist. Zwar fehlt grundsätzlich das Feststellungsinteresse, wenn der Kläger dasselbe Ziel mit einer Klage auf Leistung erreichen kann. Es besteht jedoch keine allgemeine Subsidiarität der Feststellungsklage gegenüber der Leistungsklage. Vielmehr ist eine Feststellungsklage trotz der Möglichkeit, Leistungsklage zu erheben, zulässig, wenn die Durchführung des Feststellungsverfahrens unter dem Gesichtspunkt der Prozesswirtschaftlichkeit zu einer sinnvollen und sachgemäßen Erledigung der aufgetretenen Streitpunkte führt. Dementsprechend ist in der Rechtsprechung anerkannt, dass dann, wenn eine Schadensentwicklung noch nicht abgeschlossen ist, der Kl. in vollem Umfang Feststellung der Ersatzpflicht begehren kann (st. Rspr., BGH, Urtt. v. 4.12.1986 - III ZR 205/85 -, NVwZ 1987, 733 m.w. N.; v. 21.2.1991 III ZR 204/89 -, VersR 1991, 788f. m.w. N.; Senat, Urt. v. 8.7.2003 - VI ZR 304/02 -, NJW 2003, 2827, sub II.1. m. w. N.).

[7] 2. So liegt es, wie die Revision zu Recht rügt und das Berufungsgericht verkannt hat, hier. Es hat einen Schadensersatzanspruch des K1. aus $\$ \$ 280,278$, \823 Abs. 1, \$831 Abs. 1, §249 BGB wegen der am 21.10.2002 rechtswidrig durchgeführten Kaiserschnittentbindung bejaht, nach Klageerhebung eingetretene Schäden und Zukunftsschäden für möglich gehalten und insoweit der Feststellungsklage stattgegeben. Dann aber durfte es hinsichtlich des etwaig vor Klageerhebung entstandenen (Teil-)Schadens die Feststellungsklage nicht mangels Feststellungsinteresses des Kl. abweisen.

[8] Entgegen der Ansicht der Revisionserwiderung steht dem nicht entgegen, dass einzelne Schadenspositionen bei Klageerhebung bereits bezifferbar und die diesen zugrunde liegenden Sachverhalte bereits abgeschlossen gewesen sein mögen. Ein Feststellungsantrag erfasst den gesamten dem Kl. entstandenen Schaden, auch solche Positionen, die - aus welchem Grund auch immer - nicht mit der Leistungsklage geltend gemacht und auch nicht zur Begründung des Feststellungsantrags konkretisiert wurden (vgl. Senat, Beschl. v. 26.10.2010 - VI ZB 74/08 -, NJW 2011, 615, Rdnr. 8; v. 16.4.2013 - VI ZB 50/12 -, NJW-RR 2013, 1077, Rdnr. 9). Einzelne bei Klageerhebung bereits entstandene Schadenspositionen stellen daher lediglich einen Schadensteil im obigen Sinne dar.

[9] 3. Da weitere Feststellungen nach alledem nicht erforderlich sind, kann der Senat in der Sache selbst entscheiden, \563 Abs. 3 ZPO. 